GANIT J. Bangladesh Math. Soc. (ISSN 1606-3694) 32 (2012) 37-41

\title{
STRUCTURES OF SURFACES WITH NON-ZERO CONSTANT CURVATURE
}

\author{
Mohd. Altab Hossain \\ Department of Mathematics \\ University of Rajshahi, Rajshai-6205, Bangladesh. \\ Email: al_math_bd@yahoo.com
}

Received 28.01.2012 Accepted 14.07.2012

\begin{abstract}
In this paper, surfaces of positive constant curvature and those of negative curvature are studied. In certain cases, a very few important structures of this class of surfaces have been obtained.

Keywords: Positive curvature, negative curvature, isometric mapping, psudosphere, semigeodesic parameterization, local structure.
\end{abstract}

\section{Introduction}

Investigations on properties of various types of surfaces are made in [2], [4] and [5]. In some cases, surfaces of constant curvature $K$ ( $K$ being greater than zero, equal to zero and less than zero) play important role in such type of study. The case when $K=0$, the surfaces belong to a particular class called the surfaces of vanishing curvature or flat surface, is studied recently in [1]. Almost similar study for another type of surfaces is made by the author of [3]. This paper deals with the study of those surfaces for which the constant curvature $K \neq 0$ i.e., when $K$ may be either greater than zero or, less than zero.

Now, some of the necessary definitions and preliminaries are recalled in the following. A point $p \in S$ is said to be an elliptic point, a hyperbolic point and $a$ parabolic point if $K>0, K<0$ and $K=0$ hold respectively.

Consider an embedded surface $S$ and let every $x^{i}(i=1,2,3)$ be the Cartesian coordinates of any $p \in S$. Then there are parameterizations $\underline{x}=\underline{x}\left(u^{\alpha}\right) ; \alpha=1,2$, having $u^{1}=u^{1}, u^{2}=$ const. and $u^{1}=$ const., $u^{2}=u^{2}$ as associated families of paths called the parametric net of the parameterization. If $g$ defines a differentiable tensor field with components $g_{11}=E, g_{12}=g_{21}=F, g_{22}=G$, where $E, F, G$ are the $1^{\text {st }}$ fundamental coefficients and if $\in$ is introduced in such a way that $\in(\underline{v}, \underline{w})=(\underline{N}, \underline{v}, \underline{w})$, where $\underline{v}, \underline{w} \in T_{p}(S)$ and $\underline{N}$ being the unit normal to $S$, there corresponds an anti-symmetric tensor with components given by 
$\epsilon_{\alpha \beta}=\in\left(\underline{x_{\alpha}}, x_{\beta}\right)=\left(\underline{N}, \underline{x}_{\alpha}, \underline{x}_{\beta}\right)$ so that $\epsilon_{12}=-\epsilon_{21}=[\operatorname{det}(g)]^{\frac{1}{2}}$ and $\epsilon_{11}=\epsilon_{22}=0$. Also, concepts about the gauss map or the spherical image, the Weingarten transformation and equations, $2^{\text {nd }}$ fundamental forms and magnitudes and Gaussian curvature are supposed to be known. If $b$ is the symmetric bilinear map $b: T_{p}(S) \times T_{p}(S) \rightarrow \boldsymbol{R}$ so that there associated a symmetric two-times covariant tensor at $p \in S$ with components given by $b_{\alpha \beta}=b\left(\underline{x}_{\alpha}, \underline{x}_{\beta}\right)=l \underline{x}_{\alpha} \cdot \underline{x}_{\beta}=-\underline{N}_{\alpha} \cdot \underline{x}_{\beta}=-\underline{N}_{\beta} \cdot \underline{x}_{\alpha}=\underline{N} \cdot \underline{x}_{\alpha \beta}$, then $b$ defines a tensor field with $b_{11}=L, b_{12}=b_{21}=M, b_{22}=N$. If the Weingarten transformation $l$ is defined as $l(\underline{v})=k \underline{v}, k$ being some scalar and $\underline{v}$ an eigenvector, then the corresponding eigenvalues $k$ are called the principal curvatures. Combining the above definitions, the principal curvature $k$ can be given by the equation $\operatorname{det}\left(b_{\gamma \beta}-k g_{\gamma \beta}\right)=0$ of second degree in $k$. Then (i) the sum of the two values of $k$, denoted by $H$, is called the mean curvature of $S$ at $p \in S$, and (ii) the product of the two values of $k$, denoted by $K$, is called the total curvature or Gaussian curvature of $S$ at $p \in S$. This $H$ and $K$ must satisfy $\quad k^{2}-H k+K=0, \quad H=g^{\alpha \beta} b_{\alpha \beta}=\operatorname{tace}(l), \quad$ and $K=\operatorname{det}\left(b_{\alpha \beta}\right) / \operatorname{det}\left(g_{\alpha \beta}\right)=\operatorname{det}(l)$. Simply, Gaussian curvature is termed curvature. If $K>0$, the curvature is called positive curvature, and if $K<0$ the curvature is called negative curvature.

Theorem 1.1[1] The curvature of a surface depends only on the first fundamental form of the surface.

Two surfaces $S$ and $S^{\prime}$ are said to be isometric if there exists a correspondence between their parameters such that the metric of $S$ transforms into the metric of $S^{\prime}$. The correspondence itself is called an isometry or an isometric mapping. A local parameterization $\underline{x}$ of $S$ is called semigeodesic if the first fundamental tensor satisfies the conditions $g_{11}=1, g_{12}=g_{21}=0$, w. r. to that parameterization.

In the basis of the above definitions, there is immediately the following result:

Proposition 1.2 For every point $p_{0} \in S$ and for every geodesic $\operatorname{arc} C$ through $p_{0}$, there is a semigeodesic parameterization of $\mathrm{S}$ at $p_{0}$ such that $C$ belongs to the parametric lines $u^{2}=$ const.

\section{Surfaces of Non-zero Constant Curvature}

There is already encountered the structures of surfaces of vanishing curvature in [1]. Now, in the following, structures for the surfaces with non-vanishing curvature have been made locally.

Firstly, a structure of cyclic surfaces having non-zero constant curvature can be given as follows: 
Theorem 2.1 All cyclic surfaces in $\mathbf{R}^{3}$ with non-zero constant curvature are just surface of revolution.

Proof. By following the proof of the theorem 2(i) in [3], one can easily prove this theorem.

Consider a point $p \in S$ and a semigeodesic parameterization $\underline{x}\left(u^{1}, u^{2}\right)$ at $p$. Then $g_{11}=1, \quad g_{12}=0$ and we shall also assume, as in the remark of Proposition 1.2, that $g_{22}\left(0, u^{2}\right)=1, \quad\left(\frac{\partial g_{22}}{\partial u^{1}}\right)_{u^{1}=0}=0$ é é .. (A). By means of this parameterization and the Gauss Theorema Egregium, one obtain $K=-\frac{1}{\sqrt{g_{22}}} \cdot\left(\sqrt{g_{22}}\right)_{11}$ so that if $K=$ const. the differential equation $\left(\sqrt{g_{22}}\right)_{11}+K \sqrt{g_{22}}=0$ is found. Fore $K>0$, the general solution of it has the following form:

$\sqrt{g_{22}}=c_{1}\left(u^{2}\right) \cos \left(\sqrt{K} u^{1}\right)+c_{2}\left(u^{2}\right) \sin \left(\sqrt{K} u^{1}\right)$ and if the initial data (A) are assumed, then $g_{22}=\cos ^{2}\left(\sqrt{K} u^{1}\right)$. Again, if $K<0$, the solution of the above differential equation with data (A) is $g_{22}=\cos h^{2}\left(\sqrt{-K} u^{1}\right)$.

The above information shows that the value of $K=$ const. defines completely the first fundamental form of the surface. Two surfaces of the same constant curvature have the same first fundamental forms at corresponding points if one considers local correspondences between the two surfaces defined by the equality of the semigeodesic parameters of the surfaces. Because of this fact, it can be stated as follows:

Theorem 2.2 Any two surfaces of the same constant curvature are locally isometric, and have the same intrinsic geometry.

The above theorem helps one to look for some standard models of surfaces of constant curvature. The principal curvatures at every point of a sphere are both equal to $\frac{1}{r}, r$ being the radius of the sphere. Therefore, the total curvature is $\frac{1}{r^{2}}$, and so if $K>0$, the sphere of radius $\frac{1}{\sqrt{K}}$ can be taken as the standard model of a surface of constant positive curvature. Consequently one has the following theorem:

Theorem 2.3 Every surface of constant positive curvature is locally isometric to a sphere. A semi geodesic parameterization of a sphere of radius $r$ is provided by the equation $x^{1}=r \cos u^{1} \cos u^{2}, \quad x^{2}=r \cos u^{1} \sin u^{2}, x^{3}=r \sin u^{1}$ followed by the parameter transformation $\tilde{u^{1}}=r u^{1}, \quad \tilde{u^{2}}=r u^{2}$. This suggests to look for a model of the case $K<0$ in the class of the surface of revolution. 
Consider the surface of revolution defined by

$x^{1}=h\left(u^{1}\right) \cos u^{2}, x^{2}=h\left(u^{1}\right) \sin u^{2}, x^{3}=k\left(u^{1}\right)$ é ..(B), where

$h\left(u^{1}\right)=r \exp \left(\frac{u^{1}}{r}\right), k\left(u^{1}\right)=\int_{0}^{u^{1}}\left[1-\exp \left(\frac{2 u^{1}}{r}\right)\right]^{\frac{1}{2}} d t ; r>0$. The surface (B) is termed pseudosphere. Then for the surface under invesigation one has obviously the result: $g_{11}=1, g_{12}=0, g_{22}=h^{2}$. Hence (B) is a semigeodesic parameterization, and formula (A) yields $K=-\frac{1}{r^{2}}$. So the description can be stated as the following:

Theorem 2.4 Every surface of constant negative curvature is locally isometric to a pseudosphere.

Theorem 2.5 Let $\mathrm{S}$ be a compact surface in $E^{3}$. Then there is at least one point $p \in S$ such that $K(p)>0$.

Proof. Since $S$ is compact it is bounded, and one can define $r$ as the infimum of the radii of the closed disks containing $\mathrm{S}$ with centre at the origin, then $\Sigma_{r} \cap S \neq \Phi, \Sigma_{r}$ being the sphere of radius $r$ centered at the origin. If $p \in \Sigma_{r} \cap S$, then $\Sigma_{r}$ and $\mathrm{S}$ have the same unit normal vector $\underline{N}$ at $p$. Otherwise, $\mathrm{S}$ gets out from the disk bounded by $\Sigma_{r}$ and, therefore, the same tangent plane. In this situation, if $\underline{v}$ is a common tangent of $\mathrm{S}$ and $\Sigma_{r}$, and if $\alpha$ is the plane defined by $(\underline{v}, \underline{N})$, then $\alpha$ cuts $\mathrm{S}$ and $\Sigma_{r}$ by curves whose normal curvatures exactly have the same sign. Because of the fact that $S$ is interior to $\Sigma_{r}$ and $\frac{1}{r}$ is the normal curvature of any curve on $\Sigma_{r}$, the section $S \cap \alpha$, of course, has a large curvature $k_{n}$ i.e., $\left|k_{n}\right| \geq \frac{1}{r}$. So applying this to the principal vectors $\underline{v}$ of $S$ at $p$, finally one gets $K(p)=k_{1}(p) k_{2}(p) \geq \frac{1}{r^{2}}$ so that $K(p)>0$.

\section{Conclusion}

The local geometric structures of the surfaces with non-zero curvature have been obtained completely through the theorem 2.3 and theorem 2.4 in the last section. Moreover, from the last theorem 2.5, it can be concluded that there is no complete surface in $E^{3}$ with strictly constant negative sectional curvature. 


\section{REFERENCES}

[1] Hossain M. A., On some characterizations of surfaces with constant curvature zero,Journal of Bangladesh Academy of Sciences, Vol. 36, No. 1, 33-37, 2012.

[2] Kreyszig E., Differential geometry, Dover publication inc., Mineola, New York, 1991.

[3] Lopez R., Cyclic surfaces of constant Gauss curvature, Houston Journal of Mathematics, Vol. 27, No. 4, 799-805, 2001.

[4] Pressley A., Elementary differential geometry, Springer-Verlag, London, 2001.

[5] Safi M. S., A study on a particular class of surfaces and manifolds, An M.Sc thesis in the dept. of mathematics, University of Rajshahi, Bangladesh, 2008. 